\title{
Respuesta de variedades de papa (Solanum tuberorum, L) a la aplicación de abonos orgánicos y fertilización química
}

\section{Response potato varieties (Solanum tuberosum $\mathrm{L}$ ) to fertilizer application to organic chemistry and fertilization}

\author{
${ }^{\triangle}$ Ricardo Luna Murillo${ }^{1}$, Kleber Espinosa Cunuhay ${ }^{1}$, Raúl Trávez Trávez ${ }^{1}$, Carmen Ulloa Méndez \\ Ana Espinoza Coronel ${ }^{2}$, Adriana Bejarano Albornoz ${ }^{3}$
}

${ }^{1}$ Coordinación de Investigación de la Universidad Técnica de Cotopaxi Extensión La Maná, Av. Los Almendros y Pujili. La Mana,Cotopaxi,Ecuador.ricardo.luna@utc.edu.ec; kleber.espinoza@utc.edu.ec; raul.travez@utc.edu.ec; carmen.ulloa@utc. edu.ec

${ }^{2}$ Consultoría técnica Consult_AR. Prooquia El Guayacán, Cdla La Carmita, Mz. 1, S13. consultar_ar@yahoo.es ${ }^{3}$ Unidad de Estudios a Distancia, Universidad Técnica Estatal de Quevedo, Campus Ing. Manuel Haz Álvarez, km $1.5 \mathrm{vía}$ a Santo Domingo de los Tsáchilas.EC.120501.Quevedo,Ecuador.fguevara@uteq.edu.ec; hcastillo@uteq.edu.ec; adriana. albornoz@hotmail.com

\section{Resumen}

$\mathrm{L}$ a fertilización o abonamiento de este cultivo constituye un factor importante de manejo, orientado a obtener una adecuada nutrición como fundamento para alcanzar los máximos rendimientos comerciales por unidad de superficie. El objetivo del presente estudio fue evaluar la respuesta agronómica de dos variedades de papa a la aplicación de abonos orgánicos y fertilización química. La investigación se desarrolló en la hacienda San Isidro, parroquia Mulaló, provincia Cotopaxi. La aplicación de abonos orgánicos se realizó a los 0 y 60 días y fueron gallinaza $\left(2.50 \mathrm{t} \mathrm{ha}^{-1}\right)$, estiércol bovino (10 $\left.\mathrm{tha}^{-1}\right)$ y humus de lombriz $\left(0.70 \mathrm{t} \mathrm{ha}^{-1}\right)$; los fertilizantes 10-30-10 y 15-15-15 (N, Py K, en porcentajes) en $0.38 \mathrm{t} \mathrm{ha}^{-1}$ cada uno; a los 0, 60 y 90 días, expuestas en un Diseño de Bloques Completos al Azar en arreglo factorial $2 \times 3 \times 2+$ dos testigos y tres repeticiones. Se midió altura de planta, diámetro de tubérculos, cantidad y peso de tubérculos por planta, rendimiento por parcela. Los resultados mostraron igual comportamiento en todos los tratamientos estudiados. Se concluye que la papa es un cultivo que responde favorablemente a la aplicación de abonos orgánicos o la interacción abonos orgánicos y fertilizantes químicos, representando una buena opción para reducir el uso de fertilizantes químicos.

Palabras clave: gallinaza, estiércol de ganado vacuno, humus de lombriz, tubérculo

Recibido: 25-noviembre-2014. Recibido en forma corregida: 28-septiembre-2015. Aceptado: 28-enero-2016.

Publicado como NOTA TÉCNICA en Ciencia y Tecnología 9(1): 11-16

Junio de 2016

\begin{abstract}
Gertilization or fertilization of this crop is a major

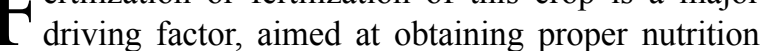
as a basis to achieve the highest commercial yields per unit area. The aim of this study was to evaluate the agronomic response of two varieties of potato to the application of organic manure and chemical fertilizers. The application of organic fertilizers was performed at 0 and 60 days; manure $\left(2.50 \mathrm{t} \mathrm{ha}^{-1}\right)$, cattle manure (10 $\left.\mathrm{t} \mathrm{ha}^{-1}\right)$ and vermicompost (0.70 $\left.\mathrm{t} \mathrm{ha}^{-1}\right) ; 10-30-10$ and 15-15-15 fertilizer (N, P and $\mathrm{K}$, in percentage) at $0.38 \mathrm{t} \mathrm{ha}^{-1}$ each; at 0,60 and 90 days, exposed on a design randomized complete block in factorial $2 \times 3 \times 2$ + two witnesses and three repetitions. The research was conducted at the Hacienda San Isidro parish Mulaló, Cotopaxi province, and plant height, diameter tubers, quantity and weight of tubers per plant, yield per plot was measured. The results showed similar behavior in all treatments studied. It is concluded that the potato is a crop that responds favorably to the application of organic fertilizers or organic fertilizers and chemical fertilizers interaction, is a good option to reduce the use of chemical fertilizers.
\end{abstract}

Key words: Chicken manure, cattle manure, vermicompost, tuber 


\section{Introducción}

$\mathrm{E}$ cultivo de papa tiene un rol protagónico en la cadena alimenticia global, fuera de los cereales, es el alimento más importante en el mundo. En el Ecuador, un total del $0.40 \%$ del territorio de uso agropecuario se dedica a la producción de papa, lo que corresponde a 49,719 ha- (Suquilanda, 2011). Esta solanácea, se ubica entre los cinco cultivos más importantes en el ámbito mundial, después del trigo, el arroz y el maíz. La producción mundial alcanzó 85860000 millones de toneladas en el 2012, siendo China el primer productor, seguido por India y Estados Unidos (FAO, 2012).

El $32.24 \%$ son productores pequeños, con unidades menores a una ha, el $29.54 \%$ producen papa como cultivo solo y el $2.7 \%$ la cultivan en asociación con otros productos. Es menester destacar que mientras menor es el predio agrícola dedicado a la papa, es mayor el número de productores con cultivo asociado. Por otra parte el $31.46 \%$ de los productores de papa tienen menos de una ha; el $75.13 \%$ son productores de menos de cinco ha. De 26,035 agricultores dedicados a la producción de papa lo hacen en una extensión de 3,627 ha, en extensiones que promedian los $1,400 \mathrm{~m}^{2}$.

El rubro de papa contribuye el $7.40 \%$ en el PIB agrícola en Ecuador, este tubérculo es sin duda una de las principales fuentes de trabajo e ingresos en región rural andina. Entre las provincias productoras se encuentra Chimborazo con mayor número de hectáreas sembradas $(10,681)$, seguida de Cotopaxi (9,672 ha); Tungurahua (7,380 ha) y Carchi $(6,179$ ha) (Devaux et al., 2010).

Las altas producciones por unidad de superficie en el cultivo implican altas extracciones de nutrimentos; las cuales dependen de varios factores, tales como las exigencias de la variedad, régimen de humedad, temperatura, producción y manejo ( Solórzano, 2001).

Al respecto, Ramírez et al. (2004) sostienen que la fertilización nitrogenada a las dosis 100 y $150 \mathrm{~kg} \cdot \mathrm{ha}^{-1}$ de N produce mayor tuberización y rendimiento. Aguilar-Acuña et at. (2003) encontraron incremento en el rendimiento de dos variedades de papa (Tollocan y Mexiquense) con aplicación de fertilización fosfatada.

El desarrollo de los cultivos demanda de una elevada aplicación de fertilizantes minerales y pesticidas, pues estos constituyen elementos básicos imprescindibles para aumentar los rendimientos agrícolas (Garcés, 2000). No obstante, se ha comprobado que el uso indiscriminado de dichos insumos químicosimplicano solo un costo elevado, sino que consuaporte se contamina el suelo, se reduce la biodiversidad, aumentan los riesgos de salinización, disminuyen considerablemente las reservas energéticas del suelo y se contaminan las aguas superficiales y subterráneas (Dierksmeier, 2007). Atendiendo a esta situación se hace necesario evaluar alternativas de fertilización para el cultivo que resulten ser más económicas a los agricultores y ambientalmente más sostenibles (Muñoz y Lucero, 2008). Especial énfasis ha cobrado la utilización de abonos orgánicos; debido fundamentalmente al papel crucial que este cumple en la nutrición vegetal y su efecto en la incorporación de determinados nutrientes a las plantas y al suelo.

Dentro de los factores que inciden en el bajo rendimiento en el cultivo de la papa es el mal empleo de fertilizantes, por consiguiente este estudio tiene como objetivo evaluar la respuesta agronómica de dos variedades de papa a la aplicación de abonos orgánicos y fertilización química.

\section{Materiales y métodos}

T a investigación se efectuó en la hacienda San Isidro de la parroquia Mulaló, cantón Latacunga, provincia de Cotopaxi, con una ubicación geográfica de $00^{\circ} 93^{\prime} 00^{\prime \prime}$ de latitud Sur, $78^{\circ} 61^{\prime} 00^{\prime \prime}$ de longitud Oeste y con una altitud de $2955 \mathrm{msnm}$, la temperatura media anual es $14^{\circ} \mathrm{C}$, la humedad relativa es $82 \%$, la precipitación $613.20 \mathrm{~mm}$, la heliofanía hora luz ${ }^{-1}$ año ${ }^{-1}$ es de 39.30 y la evaporación promedio anual 70.40.

El trabajo experimental estuvo comprendido entre los meses abril-julio, los factores en estudio fueron variedades de papa (Libertad y Rosita); abonos orgánicos (gallinaza (2.50 t $\left.\mathrm{ha}^{-1}\right)$; estiércol de bovino $\left(10 \mathrm{tha}^{-1}\right)$ y humus de lombriz $(0.70 \mathrm{t}$ $\left.\mathrm{ha}^{-1}\right)$; y fertilizantes químicos $10-30-10\left(0.38 \mathrm{t} \mathrm{ha}^{-1}\right)$ y $15-15-$ $15\left(0.38 \mathrm{t} \mathrm{ha}^{-1}\right)$. Se utilizó el diseño de bloques completos al azar en arreglo factorial $2 \times 3 \times 2$ y 2 tratamientos control, con un total de 24 tratamientos y tres repeticiones, y un total de 72 unidades experimentales.

El área de las parcelas fue de cuatro metros de ancho por tres metros de largo con un área de $1296 \mathrm{~m}^{2}$ para todo el experimento. La fertilización se realizó según las dosis de tratamientos establecidos para el cultivo de la papa (Cuadro 1). La siembra se realizó en forma manual, se colocó al fondo

Cuadro 1. Dosis y días de aplicación de abonos orgánicos y fertilizantes químicos

\begin{tabular}{lcc}
\hline \multicolumn{1}{c}{ Abonos } & Dosis $(\mathbf{k g}$ tratamiento & - \\
\hline Gallinaza, $\left(2.5\right.$ tha $\left.^{-1}\right)$ & 9 & $0-60$ \\
Estiércol de bovino, $\left(10\right.$ tha $\left.^{-1}\right)$ & 36 & $0-60$ \\
Humus de lombriz, $\left(0.70\right.$ tha $\left.^{-1}\right)$ & 2.52 & $0-60$ \\
$10-30-10\left(0.38\right.$ tha $\left.^{-1}\right)$ & 1.36 & $0-60-90$ \\
$15-15-15\left(0.38 \mathrm{tha}^{-1}\right)$ & 1.36 & $0-60-90$ \\
\hline
\end{tabular}


del surco dos tubérculos por golpe a $0.40 \mathrm{~m}$ entre ellos y a $1.00 \mathrm{~m}$ entre surcos, luego se tapó la semilla con una capa de tierra, utilizando el azadón.

Para cada variable evaluada se tomó cinco plantas al azar de la parcela útil de cada tratamiento, se midió altura desde la parte basal hasta el ápice (cm) a los 60, 90 y 120 días (momento previo a la cosecha), conteo de tubérculos, peso fresco de tubérculos $(\mathrm{g})$, diámetro de los tubérculos $(\mathrm{cm})$, peso total de la planta $(\mathrm{kg})$ y el rendimiento $\left(\mathrm{t} \cdot \mathrm{ha}^{-1}\right)$.

Se realizaron análisis de varianza y las diferencias entre medias de cada factor y variable se realizó mediante contrastes ortogonales ( $p \leq 0.05)$, es decir, comparaciones independientes de medias. Los análisis estadísticos se realizaron con el programa Statistica v. 10.0 para Windows (StatSoft, Inc., 2011).

\section{Resultados y discusión}

$\mathrm{L}$ a altura de la planta para ambas variedades en estudio solo mostró diferencias $(\mathrm{p}<0.05)$ a los 90 días, donde la variedad Libertad alcanzó la mayor altura de planta con $29.00 \mathrm{~cm}$ con respecto a la Rosita con $24.15 \mathrm{~cm}$. Se observan diferencias para los abonos y variedades respectivamente, donde los abonos orgánicos de forma general tuvieron un mejor comportamiento, alcanzando mayor altura $(30.52 \mathrm{~cm})$ al utilizar estiércol bovino (Cuadro 2). La altura promedio de la variedad Libertad a los 120 días fue de $40.45 \mathrm{~cm}$ y para los abonos el estiércol bovino con $42.56 \mathrm{~cm}$, al someter al análisis de varianza no se encontró diferencias significativas $(\mathrm{p}<0.05)$ para las variedades y los abonos.

La respuesta de las variedades de papa (Libertad y Rosita) en relación a la altura de la planta a los 90 días (Figura 1),

Cuadro 2. Efecto simple en altura de planta $(\mathrm{cm})$ en cultivo de dos variedades de papa (Solanum tuberosum var. Libertad y var. Rosita) con abonos orgánicos y fertilizantes químicos

\begin{tabular}{|c|c|c|c|}
\hline \multirow{2}{*}{ Factores } & \multicolumn{3}{|c|}{ Altura de planta (cm) } \\
\hline & 60 & 90 & 120 \\
\hline \multicolumn{4}{|l|}{ Variedades de papa } \\
\hline Libertad & $18.55 \mathrm{a}$ & $29.00 \mathrm{a}$ & $40.45 \mathrm{a}$ \\
\hline Rosita & $18.73 \mathrm{a}$ & $24.15 b$ & $38.95 \mathrm{a}$ \\
\hline \multicolumn{4}{|l|}{ Abonos orgánicos } \\
\hline Gallinaza & $18.11 \mathrm{a}$ & $27.85 \mathrm{~b}$ & $39.20 \mathrm{a}$ \\
\hline Estiércol de bovino & $18.89 \mathrm{a}$ & $30.52 \mathrm{a}$ & $42.56 \mathrm{a}$ \\
\hline Humus de lombriz & $18.62 \mathrm{a}$ & $28.62 \mathrm{ab}$ & $37.42 \mathrm{a}$ \\
\hline \multicolumn{4}{|c|}{ Fertilizantes químicos } \\
\hline $10-30-10$ & $17.95 \mathrm{a}$ & $28.05 \mathrm{ab}$ & $39.57 \mathrm{a}$ \\
\hline $15-15-15$ & $18.00 \mathrm{a}$ & $29.12 \mathrm{ab}$ & $39.74 \mathrm{a}$ \\
\hline C.V. $(\%)$ & 9.81 & 10.55 & 11.25 \\
\hline
\end{tabular}

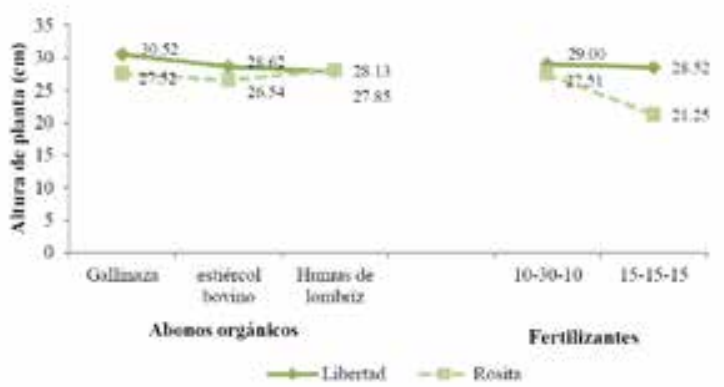

Figura 1. Altura de planta a los 90 días en la interacción variedades por abonos orgánicos $y$ variedades por fertilizantes químicos en el cultivo de dos variedades de papa (Solanum tuberosum var. Libertad y var. Rosita)

muestra un comportamiento similar al utilizar abono orgánico humus de lombriz, igualmente al aplicar fertilizantes químicos con la formulación 10-30-10.

El efecto de la combinación de abonos orgánicos y fertilizantes sobre el comportamiento de la altura de la planta a los 90 días (Figura 2), fue similar para esta variable en ambas variedades al aplicar gallinaza 15-15-15, estiércol bovino 10 30-10, humus de lombriz 10-30-10 y humus de lombriz 15-1515.

La altura de la planta en el cultivo de papa variedad Libertad en el tratamiento control fue menor en relación al promedio de alturas de los tratamientos alternativos orgánicos, debido a que alcanzaron los mayores promedios, esto permite analizar que la aplicación de abono orgánico en el suelo induce mayor absorción de nutrientes, por ende favorece el desarrollo vegetativo del cultivo, lo que no ocurre al aplicar el fertilizante químico.

Estos resultados guardan estrecha relación a lo planteado por Zamora y Rodríguez (2008) y Buchanan (1993) quienes obtuvieron valores superiores con la aplicación de la fertilización orgánica, el desarrollo vegetativo en la mayoría de los casos, fue muy superior a la fertilización química, lo que evidencia las bondades del uso de este tipo de abono para la fertilización del cultivo de la papa.

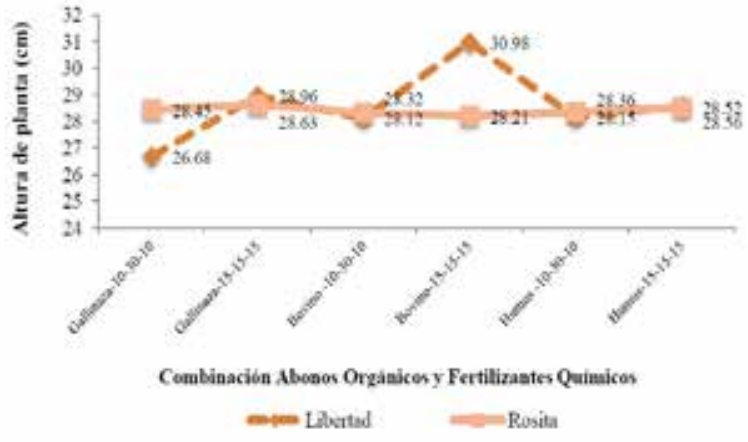

Figura 2. Altura de planta a los 90 días en la interacción variedades por abonos orgánicos por fertilizantes químicos en el cultivo de dos variedades de papa (Solanum tuberosum var. Libertad y var. Rosita) 
Los abonos orgánicos influyen en el crecimiento de las plantas, lo cual coincide con Fernández-Luqueño et al. (2010) que reportan crecimiento superior a $1 / 3$ respecto al control en altura de planta en frijol tratado con vermicompost. Resultado similar reporta Channabasanagowda et al. (2008) en trigo. Buniselli et al. (1990) reportan incrementos de peso, altura de planta, longitud de mazorca y rendimiento de grano en maíz.

Lo anterior se debe a que los ácidos húmicos presentes en los abonos orgánicos incrementan la permeabilidad de la membrana celular en plantas de papa, que a pesar de las condiciones hipertensas del medio, favorece la imbibición para solubilizar almidones y carbohidratos en la raíz. Los humatos presentes en los abonos orgánicos funcionan como regulador o promotor del crecimiento (Nardi et al., 2002), debido a los ácidos abscísico e indolacético del vermicompost (Barros et al., 2010; Ermakov et al., 2000) reportan que el ácido húmico absorbido por las células, aumenta su permeabilidad y la absorción de nutrimentos.

Los resultados obtenidos con relación a los indicadores de crecimiento anteriormente analizados indican que el abono orgánico fue efectivo bajo las condiciones de este tipo de suelo en la fase de campo, al lograrse incrementos significativos con respecto a los mismos. En tal sentido, se ha comprobado que este bioabono manifiesta su actividad biológica de manera significativa aún en bajas concentraciones en este cultivo. Los efectos positivos de estos abonos orgánicos en el crecimiento y desarrollo vegetal han demostrado favorecer el crecimiento.

En cuanto al diámetro de tubérculo el testigo de la variedad Libertad alcanzó el mayor promedio con 5.38 $\mathrm{cm}$; con respecto a los abonos, la gallinaza mostró el mejor promedio con $5.12 \mathrm{~cm}$. Respecto a la cantidad de tubérculos por planta igualmente el testigo de la variedad Libertad mostró la mayor cantidad con 9.35 tubérculos, existiendo diferencias estadísticas con el abono orgánico, gallinaza con 8.39 tubérculos. El peso total por planta estuvo dado con la cantidad de tubérculos y el peso promedio por tubérculo, encontrando a la variedad Libertad con el mayor peso por planta con $2.05 \mathrm{~kg}$ y en los abonos orgánicos la gallinaza con $2.14 \mathrm{~kg}$ (Cuadro 3).

Romero-Lima et al. (2000) en México, al evaluar diferentes fuentes orgánicas y compararlas con fuentes minerales, encontraron que cuando se aplicó la gallinaza, los requerimientos nutricionales fueron menores, se obtuvieron tubérculos de mayor calidad y se incrementaron los rendimientos. La producción fue relativamente alta para todos los tratamientos empleados en este estudio con la aplicación de abonos orgánicos y fertilizantes, lo que permite afirmar que el cultivo de papa responde favorablemente a dosis altas de materia orgánica, coincidiendo con los resultados de Pérez y Alvarado (2006), quienes, al evaluar la fertilización de papa S. phureja en los estados de Mérida y Táchira (Venezuela), encontraron en los tratamientos con altas dosis de abonamiento orgánico los mayores rendimientos cuando la fertilización estaba acompañada de un abono químico. Es conocido el
Cuadro 3. Efecto simple en diámetro de tubérculo (cm), Cantidad de tubérculos por planta y peso total por planta (kg) en cultivo de dos variedades de papa (Solanum tuberosum var. Libertad y var. Rosita) con abonos orgánicos y fertilizantes químicos

\begin{tabular}{ccc}
$\begin{array}{c}\text { Diámetro } \\
\text { de tubér- } \\
\text { culo }\end{array}$ & $\begin{array}{c}\text { Cantidad } \\
\text { tubérculos } \\
\text { por planta }\end{array}$ & $\begin{array}{c}\text { Peso } \\
\text { total de } \\
\text { planta }\end{array}$ \\
\hline
\end{tabular}

\begin{tabular}{|c|c|c|c|}
\hline \multicolumn{4}{|l|}{ Variedades de papa } \\
\hline Libertad & $5.38 \mathrm{a}$ & $9.35 \mathrm{a}$ & $2.05 \mathrm{a}$ \\
\hline Rosita & $4.56 \mathrm{a}$ & $5.91 \mathrm{~b}$ & $1.93 \mathrm{a}$ \\
\hline \multicolumn{4}{|l|}{ Abonos orgánicos } \\
\hline Gallinaza & $5.12 \mathrm{a}$ & $8.39 \mathrm{a}$ & $2.14 \mathrm{a}$ \\
\hline Estiércol de bovino & $4.94 \mathrm{a}$ & $7.94 \mathrm{a}$ & $1.94 \mathrm{a}$ \\
\hline Humus de lombriz & $4.93 \mathrm{a}$ & $6.98 \mathrm{a}$ & $2.01 \mathrm{a}$ \\
\hline \multicolumn{4}{|c|}{ Fertilizantes químicos } \\
\hline $10-30-10$ & $4.93 \mathrm{a}$ & $7.82 \mathrm{a}$ & $1.84 \mathrm{a}$ \\
\hline $15-15-15$ & $4.80 \mathrm{a}$ & $6.81 \mathrm{a}$ & $1.89 \mathrm{a}$ \\
\hline C.V. (\%) & 18.53 & 16.84 & 22.05 \\
\hline
\end{tabular}

Medias seguidas con la misma letra, no diffieren estadísticamente (Tukey, $\mathrm{p}>0.05$ )

efecto benéfico de la materia orgánica en el mejoramiento de las propiedades fisicoquímicas del suelo, ya que el cultivo de la papa reacciona favorablemente a los abonos orgánicos, porque mejora la estructura del suelo y gradualmente hay liberación de varios nutrimentos (Barrera, 2004; Mallory y Porter, 2007); de esta manera el abono orgánico constituye un suplemento ideal para los fertilizantes como el vermicompost y los biolíquidos extraídos del mismo.

Lima et al. (2000) al evaluar el rendimiento total de tubérculos en tres dosis de fertilización con abonos orgánicos y fertilizantes químicos, reportan que por cada tonelada de gallinaza aplicada, el rendimiento total se incrementa $1.468 \mathrm{t}$ por encima de las 40.752 t. Autores como Zamora y Rodríguez (2008) obtienen $32 \mathrm{t} \mathrm{ha}^{-1}$ con abonos orgánicos (estiércol de chivo). Por otra parte, Rodríguez y Ortuño (2007) obtienen de 11.53 a $15.93 \mathrm{t} \mathrm{ha}^{-1}$ con aplicación de micorrizas arbusculares en interacción con abonos orgánicos. Al respecto Corzo y Moreno (2003) mencionan que el principal elemento responsable de la movilización del almidón desde las hojas hacia el tubérculo es el $\mathrm{K}$, de tal forma que una buena disponibilidad de este nutriente es decisivo para la obtención de un alto rendimiento y calidad.

Los criterios anteriores coinciden con Carter et al. (2004), señalando que el incremento de los rendimientos en la producción de papa fertilizada con fuentes orgánicas, es debido al aumento en los contenidos de materia orgánica, lo cual incrementa la producción de tubérculos. Estos autores además atribuyen estos incrementos a mejoras en 
las propiedades físicas y biológicas de los suelos, tales como retención de humedad e incremento de la actividad biológica, así como el aporte de nutrimentos al suelo, especialmente nitrógeno.

\section{Conclusiones}

$\mathrm{L}^{2}$ a papa es un cultivo que responde favorablemente a la aplicación de abonos orgánicos o a la interacción abonos orgánicos y fertilizantes químicos. El uso de abonos orgánicos o su combinación con fertilizantes químicos, constituye una buena opción para reducir el uso de fertilizantes químicos y una alternativa transformadora de los sistemas agrícolas en modos de producción más sostenible desde el punto de vista productivo, ecológico, económico y social.

\section{Bibliografía}

Aguilar-Acuña, JL., López-Morgado, R., Núñez-Escobar, R. y Khalil, GA. 2003. Encalado y fertilización fosfatada en el cultivo de papa en un Andosol de la Sierra Veracruzana. Terra Latinoamericana. 21(3): 417-426.

Barrera, L. 2004. La fertilidad de los suelos de clima frío y la fertilidad de los cultivos. En: Fertilidad de suelos, diagnóstico y control. SCCS, Bogotá. 85 p.

Barros, DL., Pascualoto, CL., López, OF., Oliveira, AN., Eustáquio, PL., Azevedo, M., Spaccini, R., Piccolo, A. and Facanha, AR. 2010. Bioactivity of chemical transformed humic matter from vermicomposts on plant root growth. Journal of Agricultural and Food Chemistry 58: 3681-3688.

Buchanan, M. 1993. Study examines efficient use of compost. Cultivar-Santa Cruz, 11(1): 9-10.

Buniselli, M., Gigliotti, GY. and Giusquiani, YPL. 1990. Applicacione del compost da RSU in agricultura. I: effetto sulla produttivia del mais e desino dei nutrienti e dei metalli pest ani nel terreno. Agrochimica 35:13-25.

Carter, M., Sanderson, R. and Macleod, J. 2004. Influence of compost on the physical properties and organic matter fractions of a fine sandy loam throughout the cycle of a potato rotation. Canadian Journal of Soil Science. 84(2): 211-218.

Channabasanagowda, NK., Patil, B., Patil, BN. and Awaknavar, JS. 2008. Effect of organic manure on growth, seed yield and quality of wheat. Journal of Agricultural Sciences 29: 366-368.

Corzo, P. y Moreno, J. 2003. Manual de papa para productores. Corporación Colombiana de Investigación Agropecuaria, C.I. Tibaitatá, Mosquera (Colombia). $101 \mathrm{p}$.

Devaux, A., Ordinola, M., Hibon, A., Flores, R., Blajos, J. y Andrade-Piedra, J. 2010. El sector papa en la región andina. Diagnóstico y elementos para una visión estratégica (Bolivia, Ecuador y Perú). Centro Internacional de la Papa. CPAD. Lima, Perú (en línea). Consultado el 25 enero 2012. Disponible en http:// agroaldia.minag.gob.pe/biblioteca/download/pdf/ manualesboletines/papa/elsectorpapaenlaregionandina. pdf.

Dierksmeier, G. 2007. Origen y desarrollo del análisis de residuos de plaguicidas en Cuba. Fitosanidad, 11(3): $87-90$.

Ermakov, EI., Ktitorova, IN. and Skobeleva, OV. 2000. Effect of humic acid in the mechanical properties of cell walls. Russian Journal of Plant Physisology 47: 518-525.

FAO. 2012. Statistical data bases. Roma, Italy.

Fernández-Luqueño, F., Reyes, VV., Martínez, SC., Salomon, HG., Yañez, MJ., Ceballos, RJM. and Dendooven, L. 2010. Effect of different nitrogen source on plant characteristics and yield of common bean (Phaseolus vulgaris). Bioresource Technology 101: 396-403.

Garcés, N. 2000. Obtención de sustancias Bioactivas de las plantas a partir de sustancias compostadas. Curso post evento. Facultad de Agronomía. UNAH. 1-8(11): 1322.

Lima, MDR., Santos, AT., Espinosa, RG. and Cerrato, RF. 2000. Producción de papa y biomasa microbiana en suelo con abonos orgánicos y minerales. Agrociencia, 34: 261-269.

Mallory, EB. and Porter, GA. 2007. Potato yield stability under contrasting soil management strategies. Agronomy Journal 99(2): 501-510.

Muñoz, LA. y Lucero, AM. 2008. Efecto de la fertilización orgánica en el cultivo de papa criolla Solanum phureja. Agronomía Colombiana 26(2): 340-346.

Nardi, S., Pizzeghello, D., Muscolo, A. and Vianello, A. 2002. Physiological effect of humic substances on higher plants. Soil Biology and Biochemistry 34: 1527-1536.

Organización de Naciones Unidas para la Agricultura y la Alimentación (FAO). 2005. Base de Datos Estadísticos: Agricultura. [en línea] Disponible en http://faostat.fao. org/site/567/default.aspx\#ancor,consultado el 15 de febrero del 2014.

Pérez, R. y Alvarado, J. 2006. Resultados de 10 experimentos de fertilización en papa (Solanum tuberosum L.) en los estados Mérida y Táchira. 3er Congreso venezolano de la ciencia del suelo. Cedeco, Mérida. $10 \mathrm{p}$.

Ramírez, O., Cabrera, A. y Corbera, J. 2004. Fertilización nitrogenada de la papa (Solanum tuberosum L.) en la provincia de Holguin. Dosis óptima de nitrógeno. Revista Cultivos Tropicales 25(2):75-80.

Rodríguez, K. y Ortuño, N. 2007. Evaluación de micorrizas arbusculares en interacción con abonos orgánicos como coadyuvantes del crecimiento en la producción hortícola del Valle Alto de Cochabamba, Bolivia. Revista Boliviana (en línea). Consultado el 4 de Abril 
de 2007. Disponible en http://www.revistasbolivianas. org.bo/scielo.php.

Romero-Lima, M., Trinidad-Santos, A., García- Espinoza, R. y Ferrero-Cerato, R. 2000. Producción de papa y biomasa microbiana en suelos con abonos orgánicos y minerales. Agrociencias (34): 261-269.

Solórzano, PR. 2001. Manual para la fertilización de cultivos en Venezuela. Agroisleña C.A.

StatSoft Inc. 2011. Statistica. System reference. StatSoft, Inc., Tulsa, Oklahoma, USA. 1098 p.

Suquilanda, M. 2011. La producción de papa. Tierra Adentro (en línea). Consultado el 25 enero de 2012. Disponible en http://revistatierraadentro.com/index. php/agricultura/148-la-produccion-organica-de-lapapa?format $=$ pdf

Zamora, F., Tua, D. y Rodríguez, D.T. 2008. Evaluación de cinco fuentes orgánicas sobre el desarrollo vegetativo y rendimiento del cultivo de papa. Agronomía Tropical 58(3): 233-243. 